\title{
ARTICLE \\ High-dose ondansetron reduces activation of interoceptive and sensorimotor brain regions
}

\author{
Emily R. Stern ${ }^{1,2}$, Rebbia Shahab ${ }^{1,2}$, Stephanie J. Grimaldi ${ }^{3}$, Evan Leibu ${ }^{4}$, James W. Murrough ${ }^{4}$, Lazar Fleysher ${ }^{5}$, Michael K. Parides ${ }^{6}$, \\ Barbara J. Coffey ${ }^{7}$, Katherine E. Burdick ${ }^{8}$ and Wayne K. Goodman ${ }^{9}$
}

\begin{abstract}
Several psychiatric disorders involve abnormalities of interoception and associated neural circuitry centered on the insula. The development of interventions modulating interoceptive circuits could lead to novel treatment approaches for these disorders. The 5-HT3 receptor antagonist ondansetron is a good candidate for the modulation of interoceptive circuits, as 5-HT3 receptors are located abundantly on sensory pathways and ondansetron has shown some clinical utility in disorders characterized by sensory and interoceptive abnormalities. The present study tested the ability of three different doses of ondansetron to engage neural regions involved in interoception to determine the drug's utility as a therapeutic agent to target circuit abnormalities in patients. Fifty-three healthy subjects were randomized to receive a single $8-\mathrm{mg}(n=18), 16-\mathrm{mg}(n=17)$, or 24-mg $(n=18)$ dose of ondansetron and placebo before MRI scanning on separate days. Subjects performed an fMRI task previously shown to engage interoceptive circuitry in which they viewed videos depicting body movements/sensation and control videos. The results revealed a highly significant relationship between dosage and activation in bilateral insula, somatosensory and premotor regions, cingulate cortex, and temporal cortex for control but not body-focused videos. These effects were driven by a robust reduction in activation for ondansetron compared to placebo for the 24-mg group, with weaker effects for the 16-mg and 8-mg groups. In conclusion, highdose ondansetron reduces activation of several areas important for interoception, including insula and sensorimotor cortical regions. This study reveals the potential utility of this drug in modulating hyperactivity in these regions in patients.
\end{abstract}

Neuropsychopharmacology (2019) 44:390-398; https://doi.org/10.1038/s41386-018-0174-x

\section{INTRODUCTION}

The process by which the nervous system detects, integrates, and interprets sensations arising from within the body is known as interoception [1]. Several functional magnetic resonance imaging (fMRI) studies have linked the detection of body sensation to activation of an "interoceptive network" involving the insula, somatosensory cortex (inferior parietal lobule, postcentral gyrus, and paracentral lobule), premotor regions (precentral gyrus and supplementary motor area), anterior cingulate cortex (ACC), and temporal cortex [2-5]. Interoceptive awareness is associated with emotional functioning [6], and alterations of interoception have been hypothesized to be contributing factors in multiple psychiatric disorders, including obsessive compulsive disorder (OCD) [7, 8], Tourette's disorder (TD) [9, 10], eating disorders [11], anxiety and depression [12], and addiction [13]. Many of these disorders exhibit abnormalities of interoceptive circuitry, particularly in the insula [7, 10-13]. Given the wide relevance of interoceptive processes for several psychiatric disorders, there is an urgent need to develop treatments that target these processes either directly (e.g., through behavioral training [14]) or indirectly by modulating brain activity in the relevant circuitry.
One potential agent that could be efficacious for the modulation of regions involved in interoception is the 5-HT3 receptor antagonist ondansetron, which is FDA-approved for the treatment of postoperative and chemotherapy-related nausea and vomiting in total daily doses typically ranging from 8 to $24 \mathrm{mg}[15,16]$. A high density of central nervous system (CNS) 5-HT3 receptors are located in the dorsal horn of the spinal cord and brainstem areas, including trigeminal nucleus, the nucleus of the solitary tract (NTS), and area postrema [16-18], regions that are involved in ascending circuits relaying visceral and somatosensory information to higher-order processing centers in the insula, somatosensory cortex, orbitofrontal cortex, and ACC [19-21]. Tuerke et al. [22] showed that ondansetron injected directly into the posterior insula of rats led to a significant reduction in conditioned gaping -an analog of nausea-suggesting that the drug's anti-emetic effects could in principle be occurring through actions on higherorder areas involved in interoception [19, 21]. In addition to its potent anti-emetic effects, ondansetron has been used to decrease other sensory symptoms, including pruritus [23] and neuropathic pain [24], suggesting a more general role for the drug in modulating signals from the body. In the field of psychiatry, ondansetron has been used with modest success in the treatment

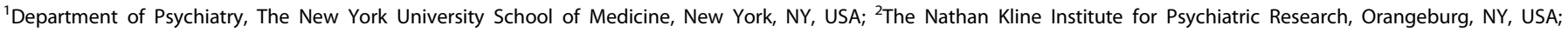

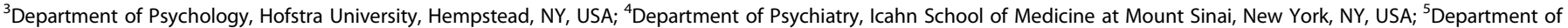

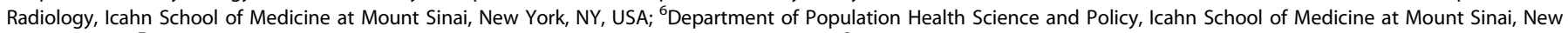

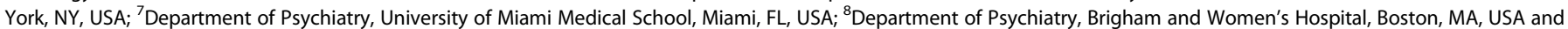
${ }^{9}$ Department of Psychiatry, Baylor College of Medicine, Houston, TX, USA

Correspondence: Emily R. Stern (emily.stern@nyumc.org)
}

Received: 9 November 2017 Revised: 22 July 2018 Accepted: 29 July 2018

Published online: 8 August 2018 


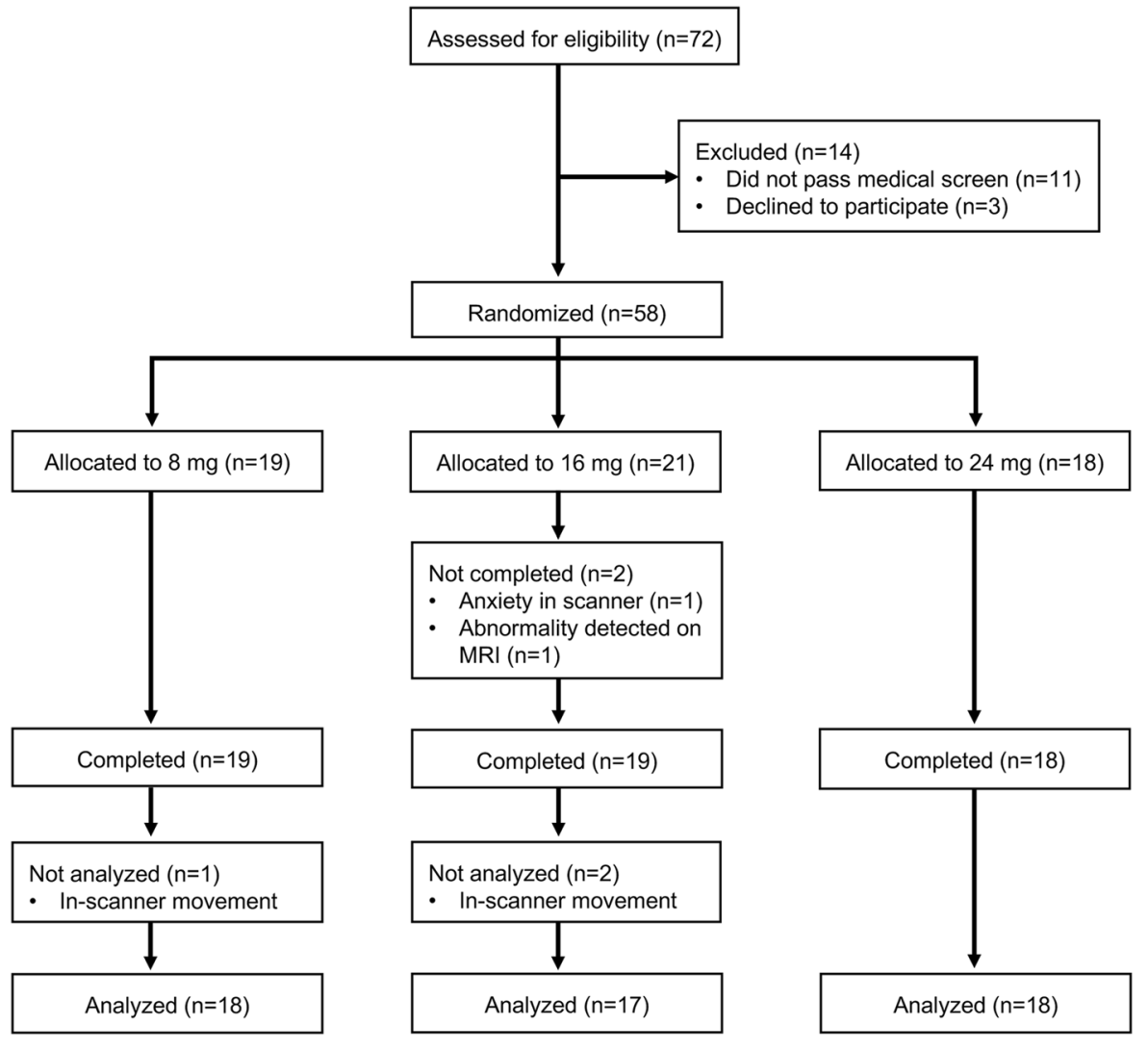

Fig. 1 Consort diagram showing participant flow in the trial

of OCD [25-27], TD [28], alcohol-dependence [29], schizophrenia [30], and bulimia [31].

To date, we are aware of only three imaging studies investigating ondansetron, two of which examined the effects of therapeutic doses ( $\geq 8 \mathrm{mg}$ ) on cerebral blood flow (CBF) in anxiety and cocainedependent patient populations, respectively $[32,33]$. Myrick et al. [34] used fMRI to examine the effects of very-low-dose ondansetron $(0.5 \mathrm{mg})$ in alcohol-dependent patients using a region-of-interest approach focusing on ventral striatum. Despite the importance of these initial studies, the findings generally showed inconsistent effects of ondansetron on brain function and, with the exception of Adinoff et al. [32], none of these studies examined the effects of ondansetron on healthy individuals, which is critical to obtain an initial measure of the neurobiological effects of the drug in the absence of disorder-related confounds. In order to determine the effects of ondansetron on interoceptive circuit activation in healthy individuals, we conducted a double-blind placebo-controlled single-dose challenge study where three different groups of healthy controls received therapeutic doses of ondansetron $(8,16$, or $24 \mathrm{mg}$ ) and placebo on two different days. Brain function during performance of an fMRI designed to elicit activation of interoceptive circuitry was measured on both days. Based on the clinical and preclinical research (described above) indicating that ondansetron reduces the intensity of certain somatosensory and viscerosensory experiences, we predicted that it would reduce activation in interoceptive circuit regions in a dose-dependent manner (with higher doses reducing activity more than lower doses).

\section{MATERIALS AND METHODS}

Subjects and procedure

Fifty-eight healthy volunteers passed medical screening and were recruited into the study over 30 months between September of 2014 and February 2017 (Fig. 1). Participants were recruited using advertisements posted on the Internet and around the Mount Sinai campus in New York City, and all participants provided written informed consent before completing any study procedures. None of them had current or previous Axis I diagnoses, as confirmed with the Mini International Neuropsychiatric Interview (M.I.N.I. [35]), and none of them were taking psychoactive medications. Five subjects were excluded from the final data set due to excessive movement in the scanner $(n=3)$ or failing to complete both sessions $(n=2)$. All of the remaining 53 subjects received ondansetron $(8,16$, or $24 \mathrm{mg})$ and placebo during different sessions $\sim 5-10$ days apart. Assignment to dose group $(8,16$, or $24 \mathrm{mg})$ and order of placebo vs. ondansetron (first vs. second session) was randomized, with 18 subjects in each of the 8-mg and 24-mg groups and 17 subjects in the 16-mg group. Sample size was determined from pilot data comparing $16 \mathrm{mg}$ of ondansetron to placebo on activation in insula and somatosensory cortex using the same fMRI task, where large effect sizes (Cohen's $d>1$ ) indicated that we would have $>95 \%$ power to detect differences between active and placebo for the mid-dose group with the current sample size. The three dose groups did not differ in age $(8 \mathrm{mg}=32.0$ years [mean] \pm 11.1 [standard deviation], $16 \mathrm{mg}=28.6 \pm 7.6$, and $24 \mathrm{mg}=32.7 \pm 11.3$ ) or years of education $(8 \mathrm{mg}=16.1 \pm 2.4 ; 16 \mathrm{mg}=16.5 \pm 2.6 ; 24 \mathrm{mg}=16.4 \pm 2.9)$. Across the final sample, there were 25 females and 28 males. Despite using random assignment to group, there was a significant difference in the proportion of females to males based on dose group (chi-square $(2)=9.1, p=0.01$ ), with $38.9 \%$ and $27.8 \%$ females in the $8-\mathrm{mg}$ and $24-\mathrm{mg}$ groups, respectively, but $76.5 \%$ females in the 16-mg group. In order to ensure any differences between dose groups were not related to this imbalance, all significant effects were followed up with post hoc analyses statistically adjusting for sex.

Placebo was matched physically to ondansetron and dose group and ordering of session was blinded to both the participant 

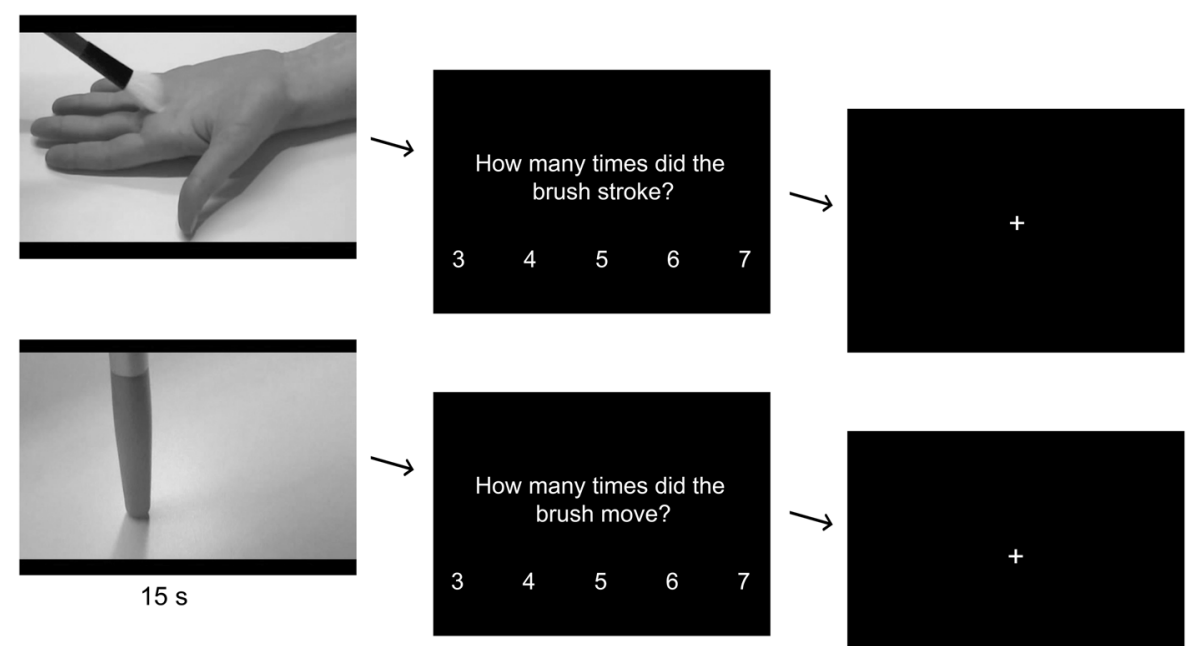

$3.5 \mathrm{~s}$

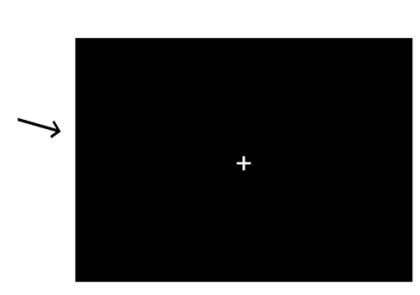

$2-6 s$

Fig. 2 Examples of body-focused videos (top) and control video (bottom) blocks in the task

and the experimenter. Participants took the dose (ondansetron, OND, or placebo, PL) in front of the experimenter $90 \mathrm{~min}$ before MRI scanning to coincide with peak effects [15]. Preparation of drug, randomization, and assignment of subjects to a group was accomplished by the Mount Sinai Research Pharmacy. Due to an administrative error made by the pharmacy, the order of placebo vs. ondansetron sessions was counterbalanced across the entire sample but not within each dose group. Specifically, for the 8-mg group, 5 subjects received placebo first and 13 received ondansetron first; for the 16-mg group, 7 subjects received placebo first and 10 received ondansetron first; and for the 24-mg group, 13 subjects received placebo first and 5 received ondansetron first. In order to ensure any differences between dose groups were not due to ordering effects, all significant effects were followed up with post hoc analyses statistically adjusting for order.

Following MRI scanning on both days, subjects completed the patient-rated inventory of side effects (PRISE [36]), which is a selfreport questionnaire that assesses the presence of various physical and mental symptoms (see Supplemental Materials and Methods and Table S1 for details). The study has been registered on clinicialtrials.gov (NCT02460341) and was approved by Mount Sinai Institutional Review Board.

Body-focused videos (BFV) task

Subjects performed an fMRI task designed to elicit activation in brain regions involved in interoception and sensorimotor processing. In the task, subjects viewed videos depicting body movements/ sensation (body-focused videos) and control videos in 15-s blocks. Previous work using similar tasks has shown that observing body touch and sensation (i.e., by viewing a video showing a brush stroking a hand) elicits activation in insula and sensorimotor regions similar to actually experiencing sensation, effects that have been attributed to a sensory "mirror neuron" system [37-41]. In contrast to tasks that engage interoceptive circuitry by asking subjects to attend to their body sensations, this approach allows for greater experimental control and does not depend as heavily on subject compliance. Three different body-focused videos depicted different scenarios: a brush stroking the underside of a hand (Fig. 2; see Supplemental Materials and Methods and Figure S1 for further rationale and description of task stimuli), a close-up of a throat while swallowing, and a medical illustration of a heart beating. The event depicted in each scenario repeated several times, with the number of repetitions varying between presentations (e.g., separate videos depict a brush stroking a hand 3, 4, or 5 times). Each body-focused video had an associated control video showing repeating non-bodyrelated events: the tip of a brush moving across a table (Fig. 2), a ball sliding through a tube, and a colored rectangle increasing and decreasing in height. As has been done in prior tasks using a similar approach [38-41], subjects were not explicitly instructed to attend (or not attend) to their own internal sensation when watching the videos, consistent with the idea that "mirror" activity is elicited automatically [38, 42,43]. Instead, subjects were simply asked to count the number of repetitions, which they reported using a 5 -point scale (3.5 s) in order to ensure that attention was focused on the videos throughout the task. After watching each video, a jittered inter-trial interval (ITI) consisting of a fixation cross was then displayed for 2-6s, after which a new video began. In total, each of the six videos (three body-focused and three control) were presented nine times (for each video there were three different numbers of repetitions, with each repetition presented three times each) for a total of 54 blocks comprised of 27 body-focused and 27 control videos.

Neuroimaging data acquisition and preprocessing

MRI scanning occurred on a Siemens 3T Skyra scanner. Functional BOLD data were acquired using a high-resolution multibandaccelerated echo-planar sequence for full brain coverage $(T R=$ $1000 \mathrm{~ms}, \mathrm{TE}=35 \mathrm{~ms}$, flip angle $=60^{\circ}, \mathrm{FOV}=228 \mathrm{~mm}, 70$ slices, 2.1-mm thickness, and acceleration factor $=7$ ). A high-resolution T1-MPRAGE structural image was acquired for co-registration $\left(\mathrm{TR}=2400 \mathrm{~ms} ; \mathrm{TE}=2.06 \mathrm{~ms} ;\right.$ flip angle $=8^{\circ} ; \mathrm{FOV}=256 \mathrm{~mm}$, 0.8-mm thickness). Preprocessing was performed using Statistical Parametric Mapping (SPM) v.12 and scripts taken from the Human Connectome Project (HCP) preprocessing pipeline [44]. The following preprocessing steps were conducted: gradient non-linearity distortion correction (HCP), realignment of functional images (SPM), fieldmap distortion correction (HCP), co-registration of functional images to a structural image, normalization to the MNI template, and spatial smoothing with a 6-mm kernel (SPM).

\section{Data analysis}

Behavioral measures. All side effects endorsed for OND and PL sessions (collapsed across dose group) are shown in Table 1. Analyses of side effects and behavioral responses during the task (ratings of the number of event repetitions) are reported in the Supplemental Materials and Methods.

Neuroimaging measures. A general linear model (SPM v.12) was used to specify regressors for all body-focused and control videos 
Table 1. Side effects for all subjects, collapsed across dose groups

\begin{tabular}{|c|c|c|c|c|}
\hline \multirow[b]{2}{*}{ Side effects } & \multicolumn{2}{|c|}{$\begin{array}{l}\text { Ondansetron }(n= \\
56)\end{array}$} & \multicolumn{2}{|c|}{ Placebo $(n=56)$} \\
\hline & No. endorsed & $\%$ & No. endorsed & $\%$ \\
\hline \multicolumn{5}{|l|}{ Gastrointestinal } \\
\hline Constipation & 0 & 0 & 1 & 2 \\
\hline Dry mouth & 6 & 11 & 9 & 16 \\
\hline Nausea/vomiting & 1 & 2 & 1 & 2 \\
\hline \multicolumn{5}{|l|}{ Heart } \\
\hline Dizziness on standing & 3 & 5 & 1 & 2 \\
\hline Palpitation & 1 & 2 & 1 & 2 \\
\hline \multicolumn{5}{|l|}{ Skin } \\
\hline Dry skin & 0 & 0 & 2 & 4 \\
\hline Increased perspiration & 1 & 2 & 0 & 0 \\
\hline Itching & 6 & 11 & 5 & 9 \\
\hline \multicolumn{5}{|l|}{ Nervous system } \\
\hline Dizziness & 7 & 13 & 3 & 5 \\
\hline Headache & 10 & 18 & 8 & 14 \\
\hline Poor coordination & 0 & 0 & 1 & 2 \\
\hline Tremors & 1 & 2 & 0 & 0 \\
\hline \multicolumn{5}{|l|}{ Eyes/ears } \\
\hline Blurred vision & 4 & 7 & 5 & 9 \\
\hline Ringing in ears & 5 & 9 & 1 & 2 \\
\hline \multicolumn{5}{|l|}{ Urinary } \\
\hline Frequent urination & 0 & 0 & 1 & 2 \\
\hline \multicolumn{5}{|l|}{ Other } \\
\hline Anxiety & 2 & 4 & 4 & 7 \\
\hline Decreased energy & 10 & 18 & 9 & 16 \\
\hline Fatigue & 20 & 36 & 21 & 38 \\
\hline General malaise & 1 & 2 & 2 & 4 \\
\hline Poor concentration & 12 & 21 & 13 & 23 \\
\hline Restlessness & 3 & 5 & 6 & 11 \\
\hline Other (need to urinate) & 0 & 0 & 1 & 2 \\
\hline
\end{tabular}

Analysis of side effects included all subjects with useable fMRI data $(n=53)$ as well as the three subjects who completed both ondansetron and placebo sessions but whose data were excluded due to in-scanner motion. There were no differences in the percentages of subjects endorsing side effects except for "ringing in the ears" (see Supplemental Materials and Methods for further details and comparisons within each dose group)

separately with duration set to block length (15 s). A regressor for the rating period was included to capture variance, with duration set equal to response time. Six motion parameters were also included to reduce error variance associated with residual movement following realignment. Scans showing movement spikes over $3-\mathrm{mm}$ translation or $2^{\circ}$ rotation were excluded and interpolated using ArtRepair [45]. At the individual subject level, four primary contrasts focused on the comparison of OND vs. PL for body-focused videos (compared to an implicit baseline consisting of ITI data points), control videos (compared to an implicit baseline), and their direct comparison (body-focused > control videos; control > body-focused videos). Group analyses investigated dose-related effects of ondansetron using multiple regressions with dosage $(8,16$, or $24 \mathrm{mg})$ as a predictor of the difference in brain activation between OND and PL sessions for each of the primary contrasts. Unless otherwise specified, correction for multiple comparisons utilized permutation testing, as suggested by Eklund et al. [46] and Cox et al. [47], with a whole-brain-corrected family-wise error rate of $p<0.05$ using threshold-free cluster enhancement [48] as implemented by palm software [49].

\section{RESULTS}

Effects of task conditions during placebo

There was broad activation in the insula, sensorimotor regions (precentral and postcentral gyri, supplementary motor area [SMA], and paracentral lobule), occipital cortex, and posterior superior temporal cortex for both body-focused and control videos when compared to an implicit baseline (Fig. 3; see Supplemental Materials and Methods and Figure S2 for activations for each video separately). Body-focused videos additionally elicited activation in bilateral superior and inferior parietal lobule/posterior postcentral gyrus, hippocampus, thalamus, orbitofrontal cortex, and left middle frontal gyrus. In the direct contrast between video types, body-focused videos showed significantly more activation in the insula, superior and inferior parietal lobule/posterior postcentral gyrus, thalamus, SMA, prefrontal cortex (including large regions of dorsolateral, inferior frontal, and orbitofrontal cortex), and lateral occipital cortex. Control videos showed more activation than body-focused videos in medial occipital cortex.

Dose-related effects of ondansetron on task activation

Contrary to expectations, there was not a significant relationship between dosage of ondansetron and differences in activation between OND and PL when subjects viewed the body-focused videos. For control videos, however, increasing dosage was associated with greater reduction in activation for OND compared to $\mathrm{PL}$ in bilateral middle and posterior insula, somatosensory cortex (postcentral gyrus), precentral gyrus, anterior cingulate cortex (ACC) and supplementary motor area (SMA), superior temporal gyrus, and left middle frontal gyrus (Table 2 and Fig. 4). Each of these clusters with the exception of the middle frontal gyrus cluster overlapped at least partially with areas that were significantly activated for the control videos vs. baseline comparison shown in Fig. 3 (bottom panel). Dose-related effects remained significant in post hoc ANOVAs adjusting for effects of order and sex $(8.8>\mathrm{F}>3.7,0.0008<p<0.04)$ for all regions except the middle frontal region, where the effect of dose on the difference between OND and PL was no longer significant in the adjusted model $\left(F_{2,41}=2.2, p=0.124\right)$.

Extracted parameter estimates revealed that these effects were driven by a somewhat large decrease in activation for OND compared to PL for the 24-mg group, a smaller decrease for OND compared to PL for the 16-mg group, and an increase for OND compared to PL for the 8-mg group (Fig. 4). Statistical comparisons within each dose group (adjusting for effects of order and sex) revealed that there was significantly less activation for OND compared to PL for all clusters in the 24-mg group $\left(F_{1,14} \geq\right.$ 7.3, $p=<0.02$ ) except for the middle frontal region, which showed a trend-level effect $\left(F_{1,14}=2.9, p=0.11\right)$ (Fig. 4). By contrast, for the 16-mg group, only the left insula cluster showed less activation for OND than $\mathrm{PL}$ at trend level $\left(\mathrm{F}_{1,13}=3.2, p=0.097\right)$. For the 8-mg group, none of the clusters showed differences between OND and PL at $p<0.10$.

In the direct comparison of dose-related effects between bodyfocused and control videos, there were no significant differences between the two video types, which was surprising given that significant dose-related effects were found for control but not bodyfocused videos. In order to further understand this finding, we examined whole-brain activations during body-focused videos at an uncorrected threshold ( $p<0.01$ using permutation testing), which did reveal an association between increasing dosage and greater reductions for OND compared to $\mathrm{PL}$ in some of the same areas as those found for control videos, including left mid insula, postcentral gyrus, and precentral gyrus. These data indicate that body-focused videos condition showed similar-but much weaker-effects as 

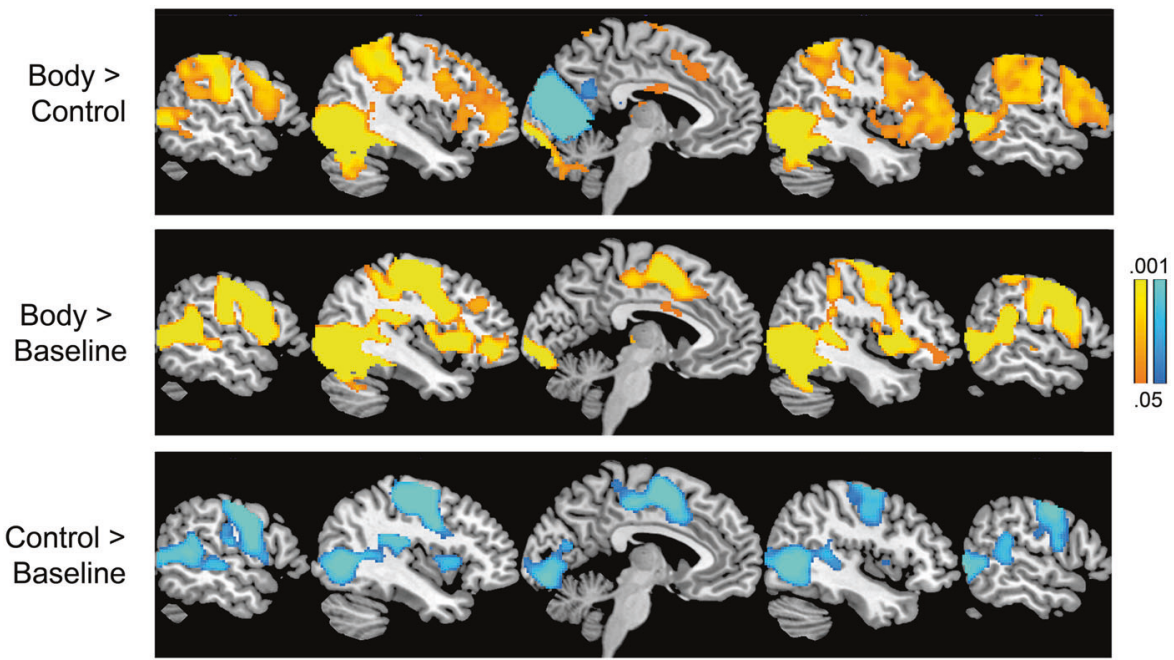

Fig. 3 Task activation during placebo session. Top row: Body-focused > control videos (warm colors) and control > body-focused videos (cool colors). Middle row: Body-focused videos $>$ baseline. Bottom row: Control videos $>$ baseline. Color bar represents whole-brain-corrected $p$ value

control videos, which may explain why the direct comparison of effects between the two video types did not yield significant differences at whole-brain-corrected thresholds. When comparing whole-brain effects between the video types using an uncorrected threshold ( $p<0.001$ using permutation testing), increasing ondansetron dosage was associated with greater reduction of activity for control than body-focused videos in left mid/posterior insula $(x=-40, y=-12, z=6$, and $k=14$, uncorrected $p=0.0002)$, SMA $(x=-10, y=-2, z=54$, and $k=5$, uncorrected $p=0.0003)$, and right precentral gyrus $(x=22, y=-18, z=58$, and $k=16$, uncorrected $p=0.0006$ ).

\section{Follow-up denoised analysis}

Because ondansetron can affect the physiology of the heart [15, $50,51]$, which in turn could impact BOLD signal $[52,53]$, we repeated first-level analysis with the inclusion of six physiological noise regressors derived from within-subjects principal component analyses (PCA) of white matter (WM) and cerebral spinal fluid (CSF) signal using the CompCor method [52] as implemented in the "Conn" tool [54], see Supplemental Materials and Methods for details. The use of principal components nuisance regressors to reduce the effects of physiological noise on gray matter BOLD signal is a well-validated noise correction approach that is widely implemented in resting-state analyses [52, 53, 55]. The results revealed significant associations between increasing dosage and greater reductions for OND compared to $\mathrm{PL}$ in those regions identified from the main analysis (Table 2) after including noise components in the model (see Table S2), indicating that the effects were not likely due to physiological noise.

\section{DISCUSSION}

The measurement of the effects of an intervention on neural targets (i.e. target engagement) is a backbone of the experimental medicine approach in psychiatry, which aims to develop novel treatments by aligning the mechanisms of an intervention with that of disease [56]. The present study used an experimental medicine approach to test the effects of three doses of ondansetron on the activation of neural targets involved in interoception and sensorimotor processing. Broadly speaking, the results confirmed our hypothesis, revealing dose-related decreases in a network involving insula, somatosensory cortex, premotor areas, ACC, and temporal cortex that were driven by significant reductions for ondansetron compared to placebo for the highest dose $(24 \mathrm{mg})$. These findings were independent of session order and sex effects; however, they were only significant during control videos and not body-focused videos. Furthermore, side effects were minimal and the majority were not different between placebo and ondansetron sessions. By determining the effects of different doses of ondansetron on neural circuit functioning, the present study has the potential to advance treatments for patient populations exhibiting abnormalities in brain regions that are modulated by the drug.

The fact that significant dose-related effects were limited to the control videos condition was unanticipated. Indeed, one might expect that effects would be found either for both video types or only for body-focused videos, when the target circuit was more actively engaged. When interrogating activity during the bodyfocused videos, we did find similar reductions related to increasing dosages of OND in some of the same areas (insula, postcentral gyrus, and precentral gyrus), but these were sufficiently weak as not to survive correction for multiple comparisons. It is unclear why the effects of ondansetron on brain function were so much stronger for control videos, although one possible explanation has to do with between-subject variability in the neural response to body-focused videos. In particular, the standard errors of the parameter estimates derived from multiple regressions examining the dose-related effect were greater for body-focused than control videos, as were the standard errors of the estimates describing the differences between the bodyfocused videos and implicit baseline for both placebo and ondansetron sessions. It is possible that there was individual variation in the degree to which the body-focused videos activated the "mirror neuron" system, which may have reduced power to detect an effect of ondansetron during these videos. Unfortunately, because we did not ask subjects to rate in the scanner how much each body-focused video drew attention to their own sensations and/or elicited a vicarious sensory response (which is thought to reflect the degree of activation of the mirror neuron sensory system, see refs. [37, 39]), our study cannot directly determine the reason for this greater variability during body-focused videos. Future work obtaining ratings and/or utilizing personalized videos could help quantify and limit interindividual variability and potentially increase power to detect the effects of ondansetron. It is also possible that physiological effects of ondansetron or other unknown drug-related factors served to increase standard errors and reduce our ability to detect effects in the body-focused videos condition. Regardless of the 

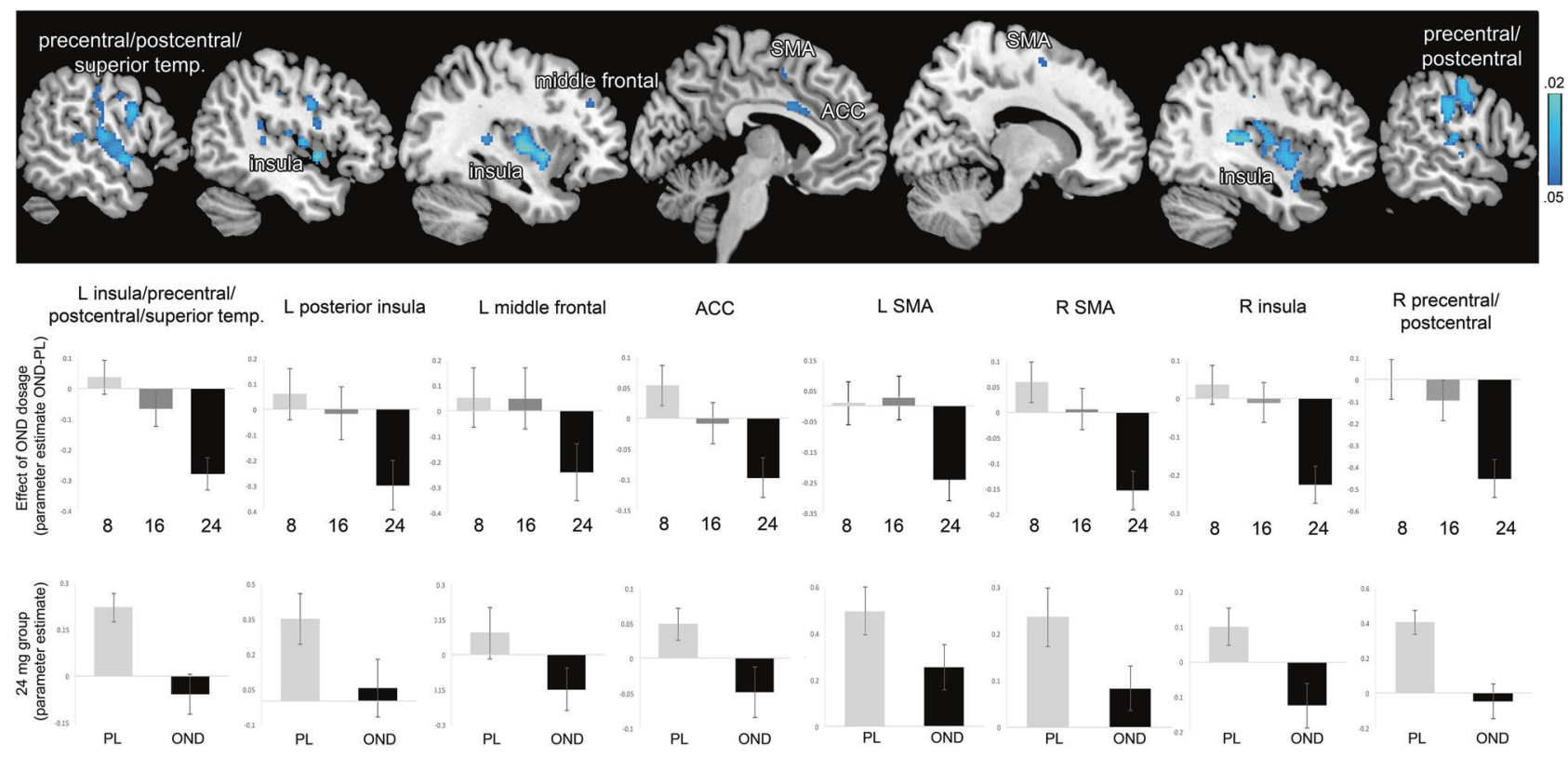

Fig. 4 Dose-related effects of ondansetron. Top row: Brain regions where increasing dosage during control videos was associated with greater reductions for ondansetron compared to placebo. Color bar represents whole-brain-corrected $p$ value. Middle row: Extracted parameter estimates showing the difference between ondansetron (OND) and placebo (PL) (negative bars indicate ondansetron less than placebo; positive bars indicate ondansetron greater than placebo) for each of the three dose groups in the regions listed in Table 2 and shown in the top row. Bottom row: Extracted parameter estimates for placebo and ondansetron (each compared to implicit baseline) for the 24-mg group for the regions listed in Table 2

reason for the more prominent effects of ondansetron in the control videos condition, these data suggest that the ability of the drug to reduce interoceptive and sensorimotor activity does not require conscious or active engagement of the circuit.

We were also surprised to find nonsignificant effects of $8 \mathrm{mg}$ of ondansetron, especially given that similar or even lower dosages show clinical efficacy in the treatment of nausea as well in several psychiatric and neurological conditions [15, 23, 25-27, 29]. It is possible that even though $8 \mathrm{mg}$ of ondansetron shows some clinical effects, the mechanism of action for this lower dose may not be occurring at the level of the cortex. Ondansetron is highly permeable to the blood-brain barrier (BBB), but is transported back out of the brain via the transmembrane protein "Pglycoprotein" and thus is measured in the brain at lower concentrations than in plasma $[57,58]$. One CNS structure not influenced by the efflux transportation of ondansetron is the area postrema, which is a circumventricular organ located outside of the BBB at the caudal edge of the medulla by the fourth ventricle [59]. This region detects circulating molecules and has bidirectional connections with brainstem structures (NTS, parabrachial nucleus) involved in interoception and somatosensory processing $[19,21,59,60]$; owing to its high concentration of 5-HT3 receptors, area postrema is thought to be involved in ondansetron's antinausea effects [59]. It is thus possible that lower doses of ondansetron work through actions on area postrema (or non-CNS systems outside of the BBB), and that higher doses (such as the 24-mg dose used in this study) are necessary to sufficiently penetrate the rest of the brain. Interestingly, Tuerke et al. [22] showed that intracranial administration of ondansetron into the posterior insula of the rat results in an anti-nausea-like response, indicating that the drug's clinical effects (at least with regard to nausea) do not require direct action on area postrema but instead may be achieved through actions at multiple access points along the interoceptive circuit. Given that the present study administered the drug systemically, it cannot be determined whether the modulation of insula and sensorimotor regions was due to direct actions on neurons in these regions or on subcortical areas that project to these regions. Ultimately, regardless of the location of ondansetron's initial action (and it is possible that it acts at multiple locations simultaneously), the present findings highlight the drug's utility for modulating the functioning of the identified circuitry.

The present work's findings are broadly consistent with a prior CBF study showing decreased blood flow in temporal and parietal cortex with a single (average) $18.5-\mathrm{mg}$ dose of IV ondansetron among patients with generalized anxiety disorder [33]. However, our high-dose group findings are in the opposite direction from those of Adinoff et al. [32], who found increased CBF in the ACC with approximately $12 \mathrm{mg}$ of IV ondansetron. Interestingly, our low-dose group also showed an increase in ACC activity with the 8-mg dose, but this finding did not survive adjustment for order and sex effects. Further work will be needed to determine whether lower-dose ondansetron has different or even opposite effects than higher-dose ondansetron on the activation of cortical regions.

Although prior studies have found that ondansetron was an effective treatment for several conditions, including pruritus [23], neuropathic pain [24], OCD [25-27], TD [28], alcohol-dependence [29], schizophrenia [30], and bulimia [31], other research has been more conflicting with regard to ondansetron's effectiveness, particularly for the treatment of anxiety [33, 61-63]. Indeed, ondansetron pretreatment has been found to accentuate physical symptoms and (nonsignificantly) increase anxiety in response to the anxiogenic drug pentagastrin in patients with panic disorder and social phobia [64]. Given these contradictory findings, the clinical implications of the present results obtained in a healthy control sample should be interpreted with caution, and future work will be needed to confirm or disconfirm ondansetron's clinical utility.

There are several limitations to this study that should be the focus of future work. We used a single-dose challenge approach and did not test the repeated effects of ondansetron on the brain or behavior. It is possible that repeated use of the drug may show different effects than single doses, and future work is aimed at 
Table 2. Brain regions showing dose-related effects of ondansetron

\begin{tabular}{|c|c|c|c|c|c|c|c|c|c|}
\hline & $\mathrm{BA}$ & $k$ & $x$ & $y$ & $z$ & pcorr & $r$ & $r_{\text {lower }}$ & $r_{\text {upper }}$ \\
\hline Superior temporal (L) & $22,41,42$ & & -38 & -36 & 8 & 0.04 & & & \\
\hline Insula (middle, posterior) (R) & 13 & 1165 & 36 & -36 & 10 & 0.02 & 0.55 & 0.33 & 0.71 \\
\hline Superior/middle temporal (R) & $22,38,41,42$ & & 60 & -16 & 10 & 0.03 & & & \\
\hline Postcentral/precentral (R) & $2,3,4,6$ & 595 & 58 & -8 & 36 & 0.03 & 0.56 & 0.33 & 0.71 \\
\hline Middle frontal (L) & 9 & 36 & -36 & 36 & 32 & 0.05 & 0.46 & 0.22 & 0.65 \\
\hline Supplementary motor area (R) & 6 & 19 & 12 & 2 & 58 & 0.05 & 0.51 & 0.27 & 0.68 \\
\hline Supplementary motor area (L) & 6 & 17 & -6 & 2 & 48 & 0.05 & 0.44 & 0.19 & 0.63 \\
\hline Insula (posterior)/superior temporal & 13 & 16 & -46 & -36 & 18 & 0.05 & 0.44 & 0.19 & 0.63 \\
\hline
\end{tabular}

Data are whole-brain corrected at a family-wise error rate of $p<0.05$ using threshold-free cluster enhancement and nonparametric permutation analysis. Positive correlation coefficients reflect greater reductions (i.e., decreased activity) for OND (compared to PL) as dosage increases

$B A$ Brodmann's areas, $k$ cluster extent, $p$ corr whole-brain-corrected $p$ value, $R$ right, $L$ left, $B$ bilateral, $r$ Pearson's correlation coefficient with $95 \%$ confidence interval bounds, coordinates are in MNI space

testing an extended dosing regimen to address this question. Furthermore, although we focused on dosages within the therapeutic range for treating nausea, lower doses have shown some efficacy in the treatment of both neurological and psychiatric disorders and our study cannot determine whether very low doses engage different neurocircuit mechanisms. Although the dose-related effect survived post hoc correction for physiological noise, it is a limitation that we do not have physiological data acquired during the task, which would have allowed us to directly examine the effects of ondansetron on physiology and relationships with brain activity. In addition, we cannot rule out the possibility that ondansetron impacted other physiological processes for which we did not correct, such as effects on vasodilation and blood pressure. Although we used a body-focused video task as a method for eliciting activity in our circuit of interest, other investigations of interoceptive circuitry have used different approaches that also successfully evoke this activity $[2,4,5,65,66]$ and which could be used in future research. Furthermore, although this study uses a hypothesis-driven taskbased approach as a first step toward characterizing the relationship between ondansetron dosage and brain function, it will be critically important for future work to interrogate effects on "resting" or baseline brain activity using non-BOLD methods such as arterial spin labeling or positron emission tomography in order to extend the applicability of these findings. Additionally, our acquisition sequence was not optimized for brainstem imaging, and the low signal in medullary areas containing an abundance of 5 -HT3 receptors limited our ability to examine the effects of ondansetron on these regions. Future work designed for brainstem and small structure imaging using higher fieldstrength magnets or specially designed sequences would complement the current findings to present a full picture of the effects of ondansetron on brain functioning.

Despite these limitations, we have found significant effects of high-dose ondansetron on activation in several areas, including insula, sensorimotor cortex, anterior cingulate, and temporal cortex, suggesting that this agent could be used to target brain functioning in patient populations showing an abnormality of these regions. It also raises the possibility that prior studies that found no effects (i.e., in OCD [67]) or mixed effects (in alcohol-dependence [29]) using very-low-dose ondansetron $(<3 \mathrm{mg})$ may wish to revisit the issue using higher doses that engage the interoceptive circuit and/or specifically measure the effects on symptoms potentially related to interoceptive processes (e.g., sensory phenomena in OCD, see ref. [7]; craving in addiction, see ref. [68]). Future work testing the effects of repeated dosing of high-dose ondansetron on brain function and interoception and body-related symptoms in patients will be needed.

\section{ACKNOWLEDGEMENTS}

We like to thank Jingwen $\mathrm{Ni}$ for her help in screening and running participants.

\section{FUNDING}

Funds for this work were provided by the National Institute of Mental Health (R21MH107589 to ERS).

\section{ADDITIONAL INFORMATION}

Supplementary Information accompanies this paper at (https://doi.org/10.1038/ s41386-018-0174-x).

Competing interests: The authors declare no competing interests.

Publisher's note: Springer Nature remains neutral with regard to jurisdictional claims in published maps and institutional affiliations.

\section{REFERENCES}

1. Khalsa SS, Adolphs R, Cameron OG, Critchley HD, Davenport PW, Feinstein JS, et al. Interoception and mental health: a roadmap. Biol Psychiatry Cogn Neurosci Neuroimaging. 2018:3:501-13.

2. Critchley HD, Wiens $S$, Rotshtein P, Ohman A, Dolan RJ. Neural systems supporting interoceptive awareness. Nat Neurosci. 2004;7:189-95.

3. Schulz SM. Neural correlates of heart-focused interoception: a functional magnetic resonance imaging meta-analysis. Philos Trans $\mathrm{R}$ Soc Lond B Biol Sci. 2016:371:20160018.

4. Simmons WK, Avery JA, Barcalow JC, Bodurka J, Drevets WC, Bellgowan P. Keeping the body in mind: insula functional organization and functional connectivity integrate interoceptive, exteroceptive, and emotional awareness. Hum Brain Mapp. 2013;34:2944-58.

5. Stern ER, Grimaldi SJ, Muratore A, Murrough J, Leibu E, Fleysher L, et al. Neural correlates of interoception: effects of interoceptive focus and relationship to dimensional measures of body awareness. Hum Brain Mapp. 2017;38:6068-82.

6. Critchley HD, Garfinkel SN. Interoception and emotion. Curr Opin Psychol. 2017;17:7-14.

7. Stern ER. Neural circuitry of interoception: new insights into anxiety and obsessive-compulsive disorders. Curr Treat Options Psychiatry. 2014;1:235-47. 
8. Yoris A, Garcia AM, Traiber L, Santamaria-Garcia H, Martorell M, Alifano F, et al. The inner world of overactive monitoring: neural markers of interoception in obsessive-compulsive disorder. Psychol Med. 2017;47:1957-70.

9. Ganos C, Garrido A, Navalpotro-Gomez I, Ricciardi L, Martino D, Edwards MJ, et al. Premonitory urge to tic in Tourette's is associated with interoceptive awareness. Mov Disord. 2015;30:1198-202.

10. Jackson SR, Parkinson A, Kim SY, Schuermann M, Eickhoff SB. On the functional anatomy of the urge-for-action. Cogn Neurosci. 2011;2:227-43.

11. Kaye $W H$, Fudge JL, Paulus $M$. New insights into symptoms and neurocircuit function of anorexia nervosa. Nat Rev Neurosci. 2009;10:573-84.

12. Paulus MP, Stein MB. Interoception in anxiety and depression. Brain Struct Funct. 2010;214:451-63.

13. Paulus MP, Stewart JL. Interoception and drug addiction. Neuropharmacology. 2014;76 Pt B:342-50.

14. Boswell JF, Farchione TJ, Sauer-Zavala S, Murray HW, Fortune MR, Barlow DH. Anxiety sensitivity and interoceptive exposure: a transdiagnostic construct and change strategy. Behav Ther. 2013;44:417-31.

15. GlaxoSmithKline. Product information: ZOFRAN tablets, oral solution, ZOFRAN ODT orally disintegrating tablets. NC: Research Triangle Park; 2013.

16. Ye JH, Ponnudurai R, Schaefer R. Ondansetron: a selective 5-HT(3) receptor antagonist and its applications in CNS-related disorders. CNS Drug Rev. 2001;7:199-213.

17. Farber L, Haus U, Spath M, Drechsler S. Physiology and pathophysiology of the 5-HT3 receptor. Scand J Rheumatol Suppl. 2004;119:2-8.

18. Tecott LH, Maricq AV, Julius D. Nervous system distribution of the serotonin 5-HT3 receptor mRNA. Proc Natl Acad Sci USA. 1993;90:1430-4.

19. Critchley HD, Harrison NA. Visceral influences on brain and behavior. Neuron. 2013;77:624-38.

20. Schachter SC, Saper CB. Vagus nerve stimulation. Epilepsia. 1998;39:677-86.

21. Craig AD. Interoception: the sense of the physiological condition of the body. Curr Opin Neurobiol. 2003;13:500-5.

22. Tuerke KJ, Limebeer CL, Fletcher PJ, Parker LA. Double dissociation between regulation of conditioned disgust and taste avoidance by serotonin availability at the $5-\mathrm{HT}(3)$ receptor in the posterior and anterior insular cortex. J Neurosci. 2012;32:13709-17.

23. Kyriakides K, Hussain SK, Hobbs GJ. Management of opioid-induced pruritus: a role for 5-HT3 antagonists? Br J Anaesth. 1999;82:439-41.

24. McCleane GJ, Suzuki R, Dickenson AH. Does a single intravenous injection of the $5 \mathrm{HT} 3$ receptor antagonist ondansetron have an analgesic effect in neuropathic pain? A double-blinded, placebo-controlled cross-over study. Anesth Analg. 2003;97:1474-8.

25. Heidari M, Zarei M, Hosseini SM, Taghvaei R, Maleki H, Tabrizi M, et al. Ondansetron or placebo in the augmentation of fluvoxamine response over 8 weeks in obsessive-compulsive disorder. Int Clin Psychopharmacol. 2014;29:344-50.

26. Pallanti S, Bernardi S, Antonini S, Singh N, Hollander E. Ondansetron augmentation in patients with obsessive-compulsive disorder who are inadequate responders to serotonin reuptake inhibitors: improvement with treatment and worsening following discontinuation. Eur Neuropsychopharmacol. 2014; 24:375-80.

27. Soltani F, Sayyah M, Feizy F, Malayeri A, Siahpoosh A, Motlagh I. A double-blind, placebo-controlled pilot study of ondansetron for patients with obsessivecompulsive disorder. Human Psychopharmacol. 2010;25:509-513.

28. Toren $\mathrm{P}$, Weizman A, Ratner S, Cohen D, Laor N. Ondansetron treatment in Tourette's disorder: a 3-week, randomized, double-blind, placebo-controlled study. J Clin Psychiatry. 2005;66:499-503.

29. Johnson BA, Roache JD, Javors MA, DiClemente CC, Cloninger CR, Prihoda TJ, et al. Ondansetron for reduction of drinking among biologically predisposed alcoholic patients: a randomized controlled trial. JAMA. 2000;284:963-71.

30. Akhondzadeh S, Mohammadi N, Noroozian M, Karamghadiri N, Ghoreishi A, Jamshidi $\mathrm{AH}$, et al. Added ondansetron for stable schizophrenia: a double blind, placebo controlled trial. Schizophr Res. 2009;107:206-12.

31. Faris PL, Kim SW, Meller WH, Goodale RL, Oakman SA, Hofbauer RD, et al. Effect of decreasing afferent vagal activity with ondansetron on symptoms of bulimia nervosa: a randomised, double-blind trial. Lancet. 2000;355:792-7.

32. Adinoff B, Devous MD, Williams MJ, Harris TS, Best SE, Dong $\mathrm{H}$, et al. Differences in regional cerebral blood flow response to a $5 \mathrm{HT} 3$ antagonist in early- and lateonset cocaine-dependent subjects. Addict Biol. 2014;19:250-61.

33. Mathew RJ, Wilson WH. Evaluation of the effects of diazepam and an experimental anti-anxiety drug on regional cerebral blood flow. Psychiatry Res. 1991;40:125-34.

34. Myrick H, Anton RF, Li X, Henderson S, Randall PK, Voronin K. Effect of naltrexone and ondansetron on alcohol cue-induced activation of the ventral striatum in alcohol-dependent people. Arch Gen Psychiatry. 2008;65:466-75.

35. Sheehan DV, Lecrubier Y, Sheehan KH, Amorim P, Janavs J, Weiller E, et al. The Mini-International Neuropsychiatric Interview (M.I.N.I.): the development and validation of a structured diagnostic psychiatric interview for DSM-IV and ICD-10. J Clin Psychiatry. 1998;59:22-33.

36. Rush AJ, Fava M, Wisniewski SR, Lavori PW, Trivedi MH, Sackeim HA, et al. Sequenced treatment alternatives to relieve depression $\left(S T A R^{*} D\right)$ : rationale and design. Control Clin Trials. 2004;25:119-42.

37. Keysers C, Gazzola V. Expanding the mirror: vicarious activity for actions, emotions, and sensations. Curr Opin Neurobiol. 2009;19:666-71.

38. Keysers C, Wicker B, Gazzola V, Anton JL, Fogassi L, Gallese V. A touching sight: SII/PV activation during the observation and experience of touch. Neuron. 2004;42:335-46.

39. Schaefer M, Heinze HJ, Rotte M. Embodied empathy for tactile events: Interindividual differences and vicarious somatosensory responses during touch observation. Neuroimage. 2012;60:952-7.

40. Schaefer M, Xu B, Flor H, Cohen LG. Effects of different viewing perspectives on somatosensory activations during observation of touch. Hum Brain Mapp. 2009;30:2722-30.

41. Blakemore SJ, Bristow D, Bird G, Frith C, Ward J. Somatosensory activations during the observation of touch and a case of vision-touch synaesthesia. Brain. 2005;128:1571-83.

42. Molnar-Szakacs I, Uddin LQ. Self-processing and the default mode network: interactions with the mirror neuron system. Front Hum Neurosci. 2013;7:571.

43. Shanton K, Goldman A. Simulation theory. Wiley Interdiscip Rev Cogn Sci. 2010;1:527-38.

44. Glasser MF, Sotiropoulos SN, Wilson JA, Coalson TS, Fischl B, Andersson JL, et al. The minimal preprocessing pipelines for the Human Connectome Project. Neurolmage. 2013;80:105-24.

45. Mazaika P, Whitfield-Gabrieli S, Reiss AL. Artifact repair for fMRI data from high motion clinical subjects. Stanford: Presentation at Organization for Human Brain Mapping; 2007.

46. Eklund A, Nichols TE, Knutsson H. Cluster failure: why fMRI inferences for spatial extent have inflated false-positive rates. Proc Natl Acad Sci USA. 2016;113:7900-5.

47. Cox RW, Chen G, Glen DR, Reynolds RC, Taylor PA. FMRI clustering in AFNI: FalsePositive Rates Redux. Brain Connectivity. 2017;7:152-171.

48. Smith SM, Nichols TE. Threshold-free cluster enhancement: addressing problems of smoothing, threshold dependence and localisation in cluster inference. Neurolmage. 2009:44:83-98.

49. Winkler AM, Ridgway GR, Webster MA, Smith SM, Nichols TE. Permutation inference for the general linear model. Neurolmage. 2014;92:381-97.

50. Dergacheva $\mathrm{O}$, Kamendi $\mathrm{H}$, Wang X, Pinol RM, Frank J, Jameson $\mathrm{H}$, et al. The role of 5-HT3 and other excitatory receptors in central cardiorespiratory responses to hypoxia: implications for sudden infant death syndrome. Pediatr Res. 2009;65:625-30.

51. Benedict CR, Arbogast R, Martin L, Patton L, Morrill B, Hahne W. Single-blind study of the effects of intravenous dolasetron mesylate versus ondansetron on electrocardiographic parameters in normal volunteers. J Cardiovasc Pharmacol. 1996;28:53-9.

52. Behzadi Y, Restom K, Liau J, Liu TT. A component based noise correction method (CompCor) for BOLD and perfusion based fMRI. Neurolmage. 2007;37:90-101.

53. Caballero-Gaudes $C$, Reynolds RC. Methods for cleaning the BOLD fMRI signal Neurolmage. 2017;154:128-49.

54. Whitfield-Gabrieli S, Nieto-Castanon A. Conn: a functional connectivity toolbox for correlated and anticorrelated brain networks. Brain Connect. 2012;2:125-41.

55. Murphy K, Birn RM, Bandettini PA. Resting-state fMRI confounds and cleanup. Neurolmage. 2013;80:349-59.

56. Insel TR. The NIMH experimental medicine initiative. World Psychiatry 2015;14:151-3.

57. Hakkarainen J, Jalkanen AJ, Kaariainen TM, Keski-Rahkonen $P$, Venalainen $T$ Hokkanen J, et al. Comparison of in vitro cell models in predicting in vivo brain entry of drugs. Int J Pharm. 2010;402:27-36.

58. Schinkel AH, Wagenaar E, Mol CA, van Deemter L. P-glycoprotein in the bloodbrain barrier of mice influences the brain penetration and pharmacological activity of many drugs. J Clin Invest. 1996;97:2517-24.

59. Price CJ, Hoyda TD, Ferguson AV. The area postrema: a brain monitor and integrator of systemic autonomic state. Neuroscientist. 2008;14:182-94.

60. Potts JT. Neural circuits controlling cardiorespiratory responses: baroreceptor and somatic afferents in the nucleus tractus solitarius. Clin Exp Pharmacol Physiol. 2002;29:103-11.

61. Olivier B, van Wijngaarden I, Soudijn W. 5-HT(3) receptor antagonists and anxiety; a preclinical and clinical review. Eur Neuropsychopharmacol. 2000;10:77-95.

62. Romach MK, Kaplan HL, Busto UE, Somer G, Sellers EM. A controlled trial of ondansetron, a 5-HT3 antagonist, in benzodiazepine discontinuation. J Clin Psychopharmacol. 1998;18:121-31.

63. Schneier FR, Garfinkel R, Kennedy B, Campeas R, Fallon B, Marshall R, et al. Ondansetron in the treatment of panic disorder. Anxiety. 1996:2:199-202. 
High-dose ondansetron reduces activation of interoceptive and...

ER Stern et al.

398

64. McCann UD, Morgan CM, Geraci M, Slate SO, Murphy DL, Post RM. Effects of the 5-HT3 antagonist, ondansetron, on the behavioral and physiological effects of pentagastrin in patients with panic disorder and social phobia. Neuropsychopharmacology. 1997;17:360-9.

65. Farb NA, Segal ZV, Anderson AK. Attentional modulation of primary interoceptive and exteroceptive cortices. Cereb Cortex. 2013;23:114-26.
66. Zaki J, Davis Jl, Ochsner KN. Overlapping activity in anterior insula during interoception and emotional experience. Neurolmage. 2012;62:493-9.

67. Transcept Pharmaceuticals I. Transcept pharmaceuticals announces that a phase 2 clinical trial of TO-2061 as adjunctive therapy for obsessive compulsive disorder did not meet primary endpoint. CA: Point Richmond; 2012.

68. Gray MA, Critchley HD. Interoceptive basis to craving. Neuron. 2007;54:183-6. 